\title{
The danger of performing meta-analysis and the impact of guidelines
}

\author{
Invited comment to: Use of antibiotic prophylaxis in elective inguinal hernia repair \\ in adults in London and south-east England: a cross-sectional survey. Aiken, A.M., et al., \\ Hernia, 2013. 10.1007/s10029-013-1061-3
}

\author{
Th. J. Aufenacker
}

Received: 15 July 2013/Accepted: 15 July 2013/Published online: 28 July 2013

(C) Springer-Verlag France 2013

Dear Editors,

I read with great interest the article by Aiken et al. [1] which is currently published in your journal and I would like to comment and elaborate on the topic.

The results of the SHAPE study are quite remarkable. This excellent study was performed in June/July 2012 with a high overall response rate of $93 \%$ of the invited surgeons. The virtual inguinal hernia repair patient for which surgeons had to decide about administrating antibiotic prophylaxis was: an ideal adult scheduled for elective surgery with no additional risk factors. In open inguinal hernia repair $87 \%$ ! of surgeons used antibiotic prophylaxis and in laparoscopic repair still $80 \%$ ! was using prophylaxis. And furthermore the hospital guidelines advised to use single dose prophylaxis in 22/28 (79 \%)! of hospitals.

Surprisingly the adherence to the local hospital guidelines was low in this study since $46 \%$ of surgeons incorrectly followed the recommendation of their own local guideline. Around $84 \%$ of surgeons believed they were doing a good thing using prophylaxis.

The conclusion of the authors of this study is that further RCTs on the topic are needed but are almost unethical because of the believed high effect of prophylaxis by surgeons.

I dare to state that no further trials are needed and will try to explain below.

This comment refers to the article available at doi:10.1007/s10029-013-1061-3.

Th. J. Aufenacker $(\bowtie)$

Department of Surgery, Rijnstate Hospital, Arnhem,

PO Box 9555, 6800 TA Arnhem, The Netherlands

e-mail: aufenacker34@ hetnet.nl; Taufenacker@rijnstate.nl
The results of this study are a big surprise to me leading to the inevitable conclusion that the European guideline on inguinal hernia repair by Simons et al. [2] published in the Hernia journal of 2009 had little impact on British surgeons at least for the topic of antibiotic prophylaxis.

This 2009 guideline [2] states on open mesh repair in low-risk patients: antibiotic prophylaxis does not significantly reduce the number of wound infections. Number needed to treat (NNT) 80. For deep infections the NNT is 352 (level of evidence 1B, Oxford centre for Evidence Based Medicine).

In endoscopic repair prophylaxis does not significantly reduce the number of wound infections with NNT $\infty$. Level of evidence $2 \mathrm{~B}$.

These conclusions lead to the grade A recommendation that in clinical settings with low rates of wound infection $(<5 \%)$, there is no indication for the routine use of antibiotic prophylaxis in elective open groin hernia repair in low-risk patients. And a grade $\mathrm{C}$ recommendation that in the presence of risk factors for wound infection based on patient (recurrence, advanced age, immunosuppression) or surgical (expected long operating times, use of drains) factors, the use of antibiotic prophylaxis should be considered.

In the current article the actual number of wound infections in the UK is not mentioned but it is relatively safe to assume that this percentage is well below $5 \%$, as it should be below $2 \%$ [3] in low-risk patients when performing clean inguinal or femoral hernia surgery lasting less than $1 \mathrm{~h}$.

The impact of the local hospital guidelines is also surprisingly low since $46 \%$ does not follow its own guidelines. Let alone that these guidelines are up to date.

The impact of the Dutch guideline on inguinal hernia published in 2003 has been analysed by de Lange et al. [4]. 
He compared data from 2001 and 2005 and demonstrated a significant impact of the guideline with a significant adherence to the protocol in 2005 regarding type of repair, the use of mesh and the number of patients treated in day care. Unfortunately, he did not look at the use of antibiotics which was reported in 2003 in the guideline with a level 4 conclusion: There is insufficient evidence to state that routine antibiotic prophylaxis is indicated in the case of the use of mesh.

\section{The problem}

I think one of the problems in this case might be the specific topic of antibiotic prophylaxis and particularly the meta-analysis.

Although the European guideline has a very clear advice, there are several authors of meta-analysis, also described by Aiken et al., with conflicting conclusions. For instance the study of $\mathrm{Li}$ [5]: in this analysis, two studies are missing and the wrong meta-analysis model is used (fixed), leading to a flawed conclusion that prophylaxis is effective. Also from the Cochrane analysis [6], the inconclusiveness prevails although they clearly state: 'Based on the results AB prophylaxis cannot be universally recommended. Neither can administration be recommended against in presence of high rates of wound infection.'

This is, I strongly believe, the big danger of metaanalysis. Meta-analysis is given much weight and influence clinical decision making without enough critical review. Many meta-analysis are incorrectly performed or are missing data and therefore lead to the wrong conclusions. When looking at the seven! meta-analyses on this current topic three out of seven (43\%!) are flawed. This is because of faults in data extraction, incomplete literature search and incorrect use of statistical methods.

Unfortunately nowadays doctors rather look at metaanalysis than good RCTs. But the doctor has to keep in mind that according to the evidence based medicine principle the key question is: "Are the results applicable to my patients". And since many meta-analyses are combinations of all trials on a topic this is usually not the case. Thus, meta-analysis is a non-perfect technique that is no substitute for a large and well-designed randomised controlled study.

So my advice for the United Kingdom would be, if you do not trust the European guideline on inguinal hernia repair, to look at a trial with a similar patient selection: for instance the largest one on this topic with 1,008 analysed patients from The Netherlands [7]. In this study, there was no benefit of antibiotic prophylaxis with an infection rate of $1.6 \%$ in the antibiotic group and $1.8 \%$ in the placebo group $[P=0.82$ $\mathrm{ARR}=0.19(-1.78$ to 1.40$)$ and NNT 520].

\section{The current evidence based verdict on antibiotic prophylaxis}

Data on non mesh repair [2] (NNT 85) also in the Cochrane analysis [6] and endoscopic surgery [2] remain clear. For this type of surgery there is no indication for antibiotic prophylaxis.

For the mesh based open repair there are currently 12 RCTs and 7 meta-analyses/reviews with conflicting results. This is mainly due to the methods of performing the analysis and the interpretation/extraction of data.

The overall meta-analysis results of the 12 RCTs have to be corrected for a large clinical diversity (inclusion criteria variations regarding diabetes and recurrent hernia) and methodological diversity (surgical variations: drain use, average surgical time, seroma aspiration, and timing of shaving) using the random model. Especially, since the results of the fixed and random analysis are conflicting. Also the percentage of wound infection in the placebo group has a large variation between 0 and $15 \%$; to correct for this broad baseline percentage of wound infections, the confidence interval must also be enlarged till $99 \%$ for the overall analysis. There can also be some comment regarding the included two studies from 2010 (Fig. 1), since they are of a possibly lower quality because of their methods of randomisation but including or excluding them in the analysis does not influence the overall conclusions. Since a formal presentation of the data used together with a systematic review is beyond the scope of this commentary, I want to point out that the same methods were follow as described in one of the earlier published meta-analysis [8].

The results of the analysis of data from 9 available studies for prevention of deep infection demonstrate an infection in $8 / 1,360(0.59 \%)$ of the placebo and $5 / 1,375$ patients $(0.36 \%)$ in the prophylaxis group with no significant benefit OR 0.63 (0.20-1.98), NNT 461.

The results of the analysis of data from all 12 RCTs demonstrate an infection of 82/1,862 (4.4\%) patients in the placebo group and 48/1,831 (2.6\%) after antibiotic prophylaxis.

Although several authors describe this as a relevant reduction in percentage, it is not a significant one OR 0.62 (0.38-1.02) (Fig. 1).

Therefore, there is no significant benefit of antibiotic prophylaxis in inguinal hernia repair (NNT 62).

A new possibility is currently available since 6 studies have a very high baseline infection percentage in the placebo group (7.0-15.4\%), therefore, another analysis can be made to estimate the usefulness of antibiotic prophylaxis in the presence of a high incidence of wound infection.

In this analysis, there is a significant benefit for the use of antibiotic prophylaxis in the presence of a high 


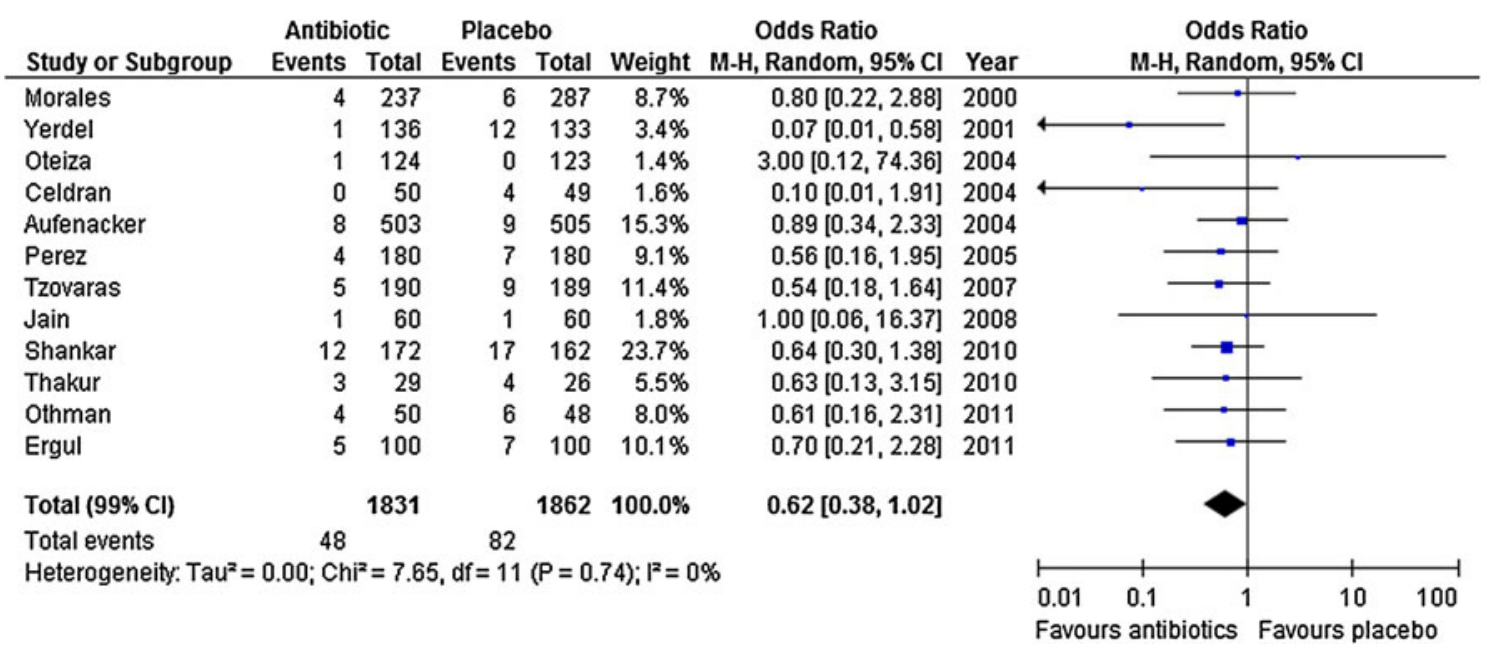

Fig. 1 Pooled data of 12 studies on the use of antibiotic prophylaxis in the prevention of wound infection after mesh inguinal repair

\begin{tabular}{|c|c|c|c|c|c|c|c|c|}
\hline Study or Subgroup & \multicolumn{2}{|c|}{ Antibiotic } & \multicolumn{2}{|c|}{ Placebo } & Weight & $\begin{array}{c}\text { Odds Ratio } \\
\text { M-H, Random, 95\% Cl }\end{array}$ & \multicolumn{2}{|c|}{ Odds Ratio } \\
\hline Morales & 4 & 237 & 6 & 287 & $0.0 \%$ & $0.80[0.22,2.88]$ & & \\
\hline Yerdel & 1 & 136 & 12 & 133 & $7.4 \%$ & $0.07[0.01,0.58]$ & & \\
\hline Celdran & 0 & 50 & 4 & 49 & $3.7 \%$ & $0.10[0.01,1.91]$ & & \\
\hline Oteiza & 1 & 124 & 0 & 123 & $0.0 \%$ & $3.00[0.12,74.36]$ & & \\
\hline Aufenacker & 8 & 503 & 9 & 505 & $0.0 \%$ & $0.89[0.34,2.33]$ & & \\
\hline Perez & 4 & 180 & 7 & 180 & $0.0 \%$ & $0.56[0.16,1.95]$ & & \\
\hline Tzovaras & 5 & 190 & 9 & 189 & $0.0 \%$ & $0.54[0.18,1.64]$ & & \\
\hline Jain & 1 & 60 & 1 & 60 & $0.0 \%$ & $1.00[0.06,16.37]$ & & \\
\hline Ergul & 5 & 100 & 7 & 100 & $20.3 \%$ & $0.70[0.21,2.28]$ & & \\
\hline Othman & 4 & 50 & 6 & 48 & $16.5 \%$ & $0.61[0.16,2.31]$ & $\pi$ & \\
\hline Shankar & 12 & 172 & 17 & 162 & $40.3 \%$ & $0.64[0.30,1.38]$ & 一 & - \\
\hline Thakur & 3 & 29 & 4 & 26 & $11.8 \%$ & $0.63[0.13,3.15]$ & & \\
\hline Total $(95 \% \mathrm{Cl})$ & & 537 & & 518 & $100.0 \%$ & $0.51[0.29,0.91]$ & & \\
\hline Total events & 25 & & 50 & & & & & \\
\hline $\begin{array}{l}\text { Heterogeneity: } \mathrm{Tau}^{2}= \\
\text { Test for overall effect: }\end{array}$ & $\begin{array}{l}.06 ; \mathrm{Chi}^{2} \\
=2.27(\mathrm{P}\end{array}$ & $\begin{array}{l}=5.59, \\
=0.02\end{array}$ & $=5(P$ & 3 & ${ }^{2}=11 \%$ & & $\begin{array}{ll}0.01 & 0.1 \\
\text { Favours } & 1\end{array}$ & $\begin{array}{lr}1 & 10 \\
\text { Favours pla }\end{array}$ \\
\hline
\end{tabular}

Fig. 2 Pooled data of 6 studies on the use of antibiotic prophylaxis in the prevention of wound infection in centres with a high incidence ( $>7 \%$ ) of wound infection after mesh inguinal repair

incidence of wound infections. OR 0.51 (0.29-0.91), NNT 22 (Fig. 2).

In the presence of a low incidence of wound infections $(0.8-4.7 \%$, the other 6 studies) there is of course no evidence of benefit from antibiotic prophylaxis with an OR $0.74(0.43-1.27)$ and NNT 170.

\section{Conclusion}

In open mesh repair in low-risk patients and with a low incidence of wound infection antibiotic prophylaxis does not significantly reduce the number of wound infections, NNT 170.
In the presence of a high incidence of wound infection $(>5 \%)$, there is a significant benefit of antibiotic prophylaxis, NNT 22.

Therefore in clinical settings with low rates of wound infection, there is no indication for the routine use of antibiotic prophylaxis in elective groin hernia repair in low-risk patients.

In institutions with high rates of wound infection (>4-5\%), the use of antibiotic prophylaxis should be considered. Furthermore in these institutions the general risk factors influencing wound infections should be checked [3]. Especially when for instance even in trials on wound infection shaving on the day before surgery and seroma aspirations are still performed. 


\section{Conflict of interest None.}

\section{References}

1. Aiken AM et al (2013) Use of antibiotic prophylaxis in elective inguinal hernia repair in adults in London and south-east England: a cross-sectional survey. Hernia (epub ahead of print)

2. Simons MP et al (2009) European Hernia Society guidelines on the treatment of inguinal hernia in adult patients. Hernia 13(4):343-403

3. Mangram AJ et al (1999) Guideline for prevention or surgical site infection, 1999. Infect Control Hosp Epidemiol 20:247-280

4. de Lange DH et al (2010) Inguinal hernia surgery in The Netherlands: are patients treated according to the guidelines? Hernia 14(2):143-148
5. Li JF et al (2012) Meta-analysis of the effectiveness of prophylactic antibiotics in the prevention of postoperative complications after tension-free hernioplasty. Can J Surg 55(1):27-32

6. Sanchez-Manuel FJ, Lozano-Garcia J, Seco-Gil JL (2012) Antibiotic prophylaxis for hernia repair. Cochrane Database Syst Rev 2:CD003769

7. Aufenacker TJ et al (2004) The role of antibiotic prophylaxis in prevention of wound infection after Lichtenstein open mesh repair of primary inguinal hernia: a multicenter double-blind randomized controlled trial. Ann Surg 240(6):955-960

8. Aufenacker TJ et al (2006) Systematic review and meta-analysis of the effectiveness of antibiotic prophylaxis in prevention of wound infection after mesh repair of abdominal wall hernia. Br J Surg 93(1):5-10 